\title{
Die automatische Generierung von Grenzliniendateien der Schweiz
}

\section{Einleitung}

In den letzten zwei Jahrzehnten sind Computermethoden entwickelt worden, die auch in der geographischen und kartographischen Forschung und Praxis Eingang gefunden haben. Neben den rechnerischen Möglichkeiten bietet die neue Technik Mittel für die graphische Datenverarbeitung und deren Verwendung in der Analyse und Darstellung räumlicher Phänomene.

Diese neuen Medien erlauben die automatische Ausführung traditioneller Verfahren, ermöglichen aber auch neue Methodologien und Strategien für geographische Analysen und die kartographische Reproduktion. Daten werden heute nicht mehr nur für die Reproduktion einer speziellen Karte erhoben und ausgewertet; sie werden vielmehr in Datenbanken räumlicher Information (in sog. Geographischen Informationssystemen) aufgesammelt, die dann für vielfältige Analyse- und Kartierzwecke zur Verfügung stehen. Solche Geographische Informationssysteme (vgl. BRASSEL 1983) setzen als Grundkomponenten eine Datenverarbeitungsanlage, eine Datenbasis und eine Bibliothek verschiedener Manipulations- und Anwendungsprogramme voraus.

Hardware, Software und Daten sind also notwendige Grundelemente für den Betrieb von geographischen Informationssystemen. Während die Hardware und die Systemsoftware durch eine wachsende Computerindustrie entwickelt werden, liegt es an den Anwendern der geographischen Datenverarbeitung, zur Entwicklung von speziellen Anwendungsprogrammen beizutragen und spezifische räumliche Dateien aufzusammeln.

In der vorliegenden Arbeit wird ein System zur Erzeugung von räumlichen Datenbasen vorgestellt. Der Anstoß dazu ging von einem Projekt des Nationalen Forschungsprogrammes "Regionalprobleme» des Schweizerischen Nationalfonds aus, für das eine Reihe von thematischen Karten auf der Basis von Bezirken, Planungsregionen und MS-Regionen (1) der Schweiz zu erstellen war (2).

Bis heute besteht keine topologisch einwandfreie Grenzliniendatei der Gemeinden der Schweiz, die sich als Grundlage für thematische Kartenprogramme eignen würde. Die Neuaufnahme der Umrisse der
3029 Gemeinden der Schweiz durch Digitalisieren ist sehr aufwendig. Dazu sind flächendeckende, einheitliche Kartenunterlagen gewünschter Genauigkeit erforderlich, sowie präzise Digitalisiergeräte und Programmpakete, die die Datenaufnahme und Fehlerkorrektur unterstützen.

Aufgrund einer Beurteilung von Ausrüstung, Zeitbedarf, Verfügbarkeit von Software und Personal entschloß man sich, ein Softwaresystem aufzubauen, das Gemeinde- und andere Grenzen aus der Flächendatei des Informationsrasters der Schweiz ableiten kann (3). Dieses System sollte darüber hinaus allgemein für den Aufbau von Grenzliniendateien aus Rasterdateien verwendbar sein.

Das Informationsraster enthält in der sogenannten Flächendatei rasterweise registrierte Informationen über das Gesamtgebiet der Schweiz (Flächeneinheit: 1 Hektare). Das Merkmal "Gemeindezugehörigkeit» speichert für jede Hektare der Schweiz einen numerischen Gemeindecode. Durch Vergleich benachbarter Zellen können nun in diesem Raster die Gemeindegranzen gefunden werden. Die erzeugten Umrißdaten weisen zunächst verschiedene Unzulänglichkeiten auf, die durch geeignete Maßnahmen korrigiert werden müssen. Eine detaillierte Darstellung der technischen Einzelschritte und speziellen Probleme findet sich in HERzoG et al. (1983). Hier sollen die wesentlichen Systemelemente im Überblick dargestellt werden.

\section{Begriffe}

Die Datenbasen geographischer Informationssysteme können in die beiden Hauptgruppen geometrische Daten (Koordinatenbeschreibung) und Deskriptordaten (Themadaten) eingeteilt werden (vgl. ICA 1980). Geometrische Daten speichern die räumlichen Attribute geographischer Phänomene, wie z. B. den Verlauf von Grenzen politischer Einheiten. Deskriptordaten geben thematische Charakteristiken räumlicher Ein-

Adrian Herzog, Kurt Brassel, Matthias Bopp und Guido Dorigo, Geographisches Institut der Universität Zürich, Winterthurerstr. 190, 8057 Zürich 


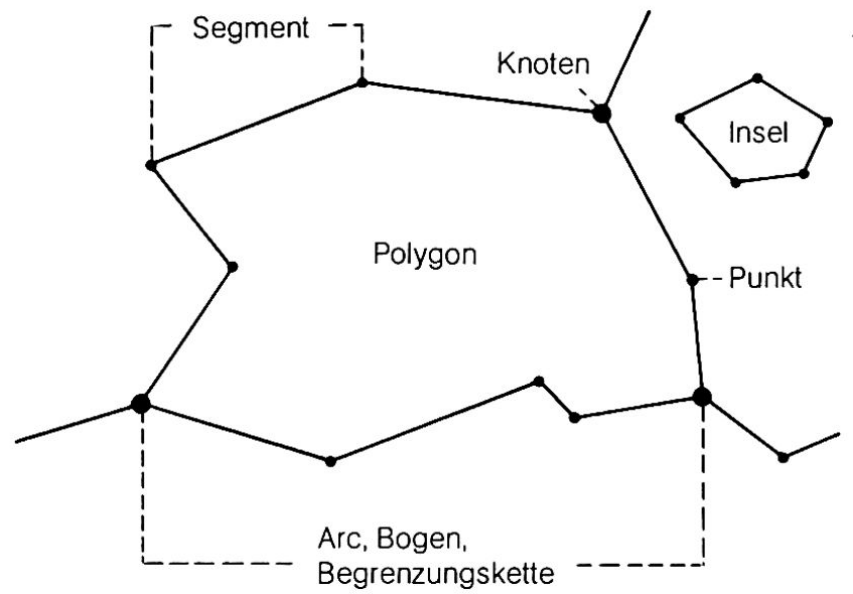

Abb. 1: Topologische Bezeichnungen
Direktzugriff-Dateien umgewandelt. Beide Dateien werden anschließend interaktiv am Bildschirmterminal bearbeitet (Fehlerkorrektur, Seenklassifikation u. a.). Nach einer allfälligen Zusammenfassung der Gemeinden in Regionen wird die Seendatei der Gebietsdatei überlagert. In einem anschließenden Schritt werden die Grenzen jeder Gebietseinheit aus diesem kombinierten Hektarraster herausgelesen und gezeichnet. Kern des Programms ist ein Verfahren, das den Grenzlinien zuverlässig folgt und Knotenpunkte und Nachbarn eindeutig erkennt. Ist der Grenzlinienverlauf unbefriedigend, können allfällige Fehler in den Hektarrasterdateien korrigiert werden. Andernfalls werden die Grenzvektoren weiterverarbeitet: Die treppenförmigen Grenzlinien werden heiten wieder. Sie müssen innerhalb der Informationssysteme mit der zugehörigen geometrischen Information verknüpft werden. Abb. 1 illustriert einige Begriffe geometrischer Grundeinheiten, die im folgenden verwendet werden (vgl. auch MONMONIER 1982, 152). Als Zielsetzung dieses Projekts wurde das Generieren von «Umrißlinien» administrativer Einheiten wie Gemeinden, Bezirken und Planungsregionen genannt. Nun besitzen zwei aneinandergrenzende Einheiten je ein gemeinsames Grenzlinienstück, das als Arc, Grenzlinienkette oder Grenzbogen bezeichnet wird (PEUCKER und CHRISMAN 1975). Grenzlinienketten stellen die elementaren linearen Bestandteile eines polygonalen Netzes von Gebietsgrenzen dar. Diese können einzeln manipuliert und generalisiert werden. Arcs werden beidseitig begrenzt durch Knoten, Punkte, an denen drei oder mehrere Grenzlinien zusammenstoßen. Die einzelnen Arcs bestehen aus einer Reihe von (kartographischen) Punkten. Die Strecke zwischen zwei aufeinanderfolgenden Punkten wird als Segment bezeichnet. Eine einzelne Gebietseinheit besteht aus einem oder mehreren Polygonen, die je durch eine Umrißkette (Polygonzug) begrenzt werden. Jede Umrißkette setzt sich aus mehreren Arcs zusammen; Inseln heißen Flächen, die von nur einer Grenzlinienkette umschlossen werden.

\section{Der Gesamtablauf im Überblick}

Abbildung 2 gibt einen Überblick über die Arbeitsschritte, die von den Hektarrasterinformationen zu fertigen Computerkarten führen.

Die Ausgangsinformation beschreibt zum einen die Gemeindezugehörigkeit jeder Hektarzelle, zum andern gibt sie Aufschluß über das Vorhandensein von Seen. Die Seeninformation wurde aus dem Merkmal «Bodennutzung» des Informationsrasters entnommen. Diese beiden Dateien werden zunächst in zwei

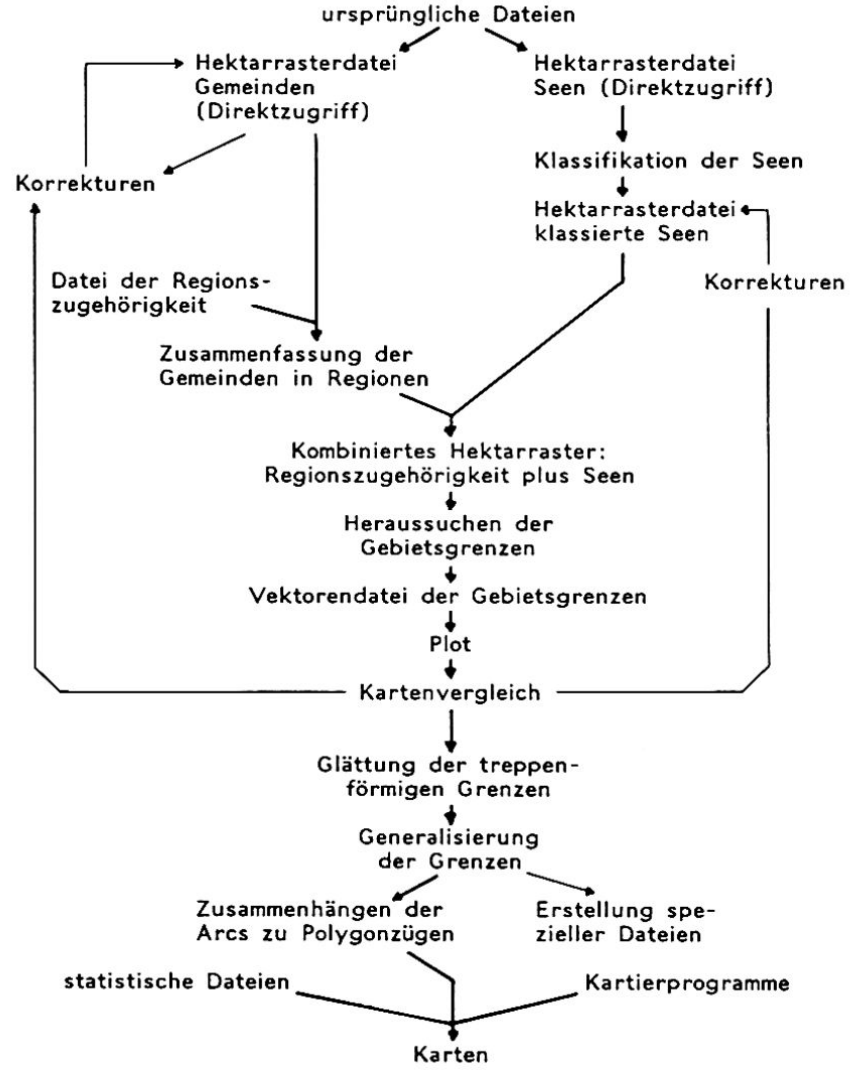

Abb. 2: Allgemeines Ablaufschema

zuerst geglättet und anschließend nach Bedarf generalisiert. Da viele Kartierprogramme die Gebietsgrenzen in Form von geschlossenen Polygonzügen erwarten, müssen diese aus den einzelnen Grenzketten aufgebaut werden. Die resultierenden Polygondateien erlauben es nun, beliebige statistische Daten mit geeigneter Software zu kartieren. Die einzelnen Schritte werden in den folgenden Kapiteln detaillierter besprochen. 


\section{Datenorganisation}

Die Flächendatei des schweizerischen Informationsrasters (kurz: Hektarraster) speichert gegenwärtig 14 Merkmale für jede der ca. 4 Millionen Hektaren der Schweiz. Die qualitativen Merkmale (wie die hier verwendeten Merkmale "Gemeindezugehörigkeit» und "Bodennutzung») speichern für jede Rasterzelle die dominante Kategorie. Um den Programmen einen möglichst schnellen Zugriff auf das Hektarraster zu ermöglichen, wurde aus der ursprünglich vom Bundesamt für Statistik gelieferten Datei für die beiden Merkmale je eine Direktzugriff-Datei erstellt, in der die Schweiz in eine Matrix von 3522 Kolonnen und 2212 Zeilen à je $100 \mathrm{~m}$ Breite aufgeteilt ist.

Das Produkt der Umwandlungen ist eine Vektorenda$t e i$, die die Grenzen aller Gebietseinheiten in Form von Umrißketten, d. h. als Folge von (x, y)-Koordinaten der Grenzpunkte beschreibt.

\section{Das Raster-Editierprogramm}

In einer Rasterdatei können auf relativ einfache Art und Weise Korrekturen vorgenommen werden. Bei Gebietsänderungen, Gebietszusammenlegungen oder -aufteilungen können direkt einzelne Rasterzellen geändert werden. Das vorgestellte System versucht deshalb, Veränderungen von Grenzverläufen in den Rasterdateien zu korrigieren und die Bearbeitung der Umrißlinien auf das Glätten und Generalisieren zu beschränken. Kompliziertere Manipulationen an der Vektorendatei fallen damit dahin. Für ein effizientes Korrigieren ist ein intelligentes graphisches Rasterfarbterminal notwendig, an dem interaktiv Korrekturen vorgenommen werden können. Dem Geographischen Institut der Universität Zürich steht für diese Aufgabe ein Farbterminal (AED 512) zur Verfügung, das an den Großcomputer der Universität (IBM 3033) angeschlossen ist.

Das Korrigieren des Hektarrasters bedingt einen mehrstufigen Datentransfer: Als permanentes Speichermedium dient ein Magnetband (tape). Von dort wird die Rastermatrix mit einem einfachen Dateikopierprogramm auf Magnetplatte (disk) kopiert. Das Korrekturprogramm liest nun Teile dieser Datei, behält diesen Ausschnitt in Form einer Matrix im Hauptspeicher des Rechners und stellt ihn auf dem Bildschirm dar. Jede Gemeinde, bzw. jede Seenkategorie, wird mit einer eigenen Farbe dargestellt, die ihr durch das Programm zugeordnet wird. Die interaktive Bearbeitung geschieht mit Hilfe eines Fadenkreuzes (cursor), das mit einem Steuerknüppel (joystick) auf dem Bildschirm bewegt werden kann. Änderungen werden mit dem Cursor auf dem Bildschirm und gleichzeitig im Hauptspeicher vorgenommen. Nach Abschluß der Korrekturarbeiten kann das Programm den überarbeiteten Ausschnitt auf Magnetplatte übertragen. Die korrigierte Version des Hektarrasters wird nach Beendigung der Arbeit wieder auf Magnetband abgespeichert.

Das Editierprogramm ist so konzipiert, daß ein der Programmierung unkundiger Benutzer damit nach kurzer Einführung arbeiten kann. Es wird eine MenuTechnik verwendet, die es erlaubt, Korrekturen verschiedenster Art vorzunehmen, wobei auf allfällige Fehler hingewiesen wird.

Mit diesem Raster-Editierprogramm wurden folgende Korrekturen vorgenommen:

$$
\begin{aligned}
& \text { - Korrekturen der Gemeindezugehörigkeit } \\
& \text { - Beseitigung von Kartenfehlern } \\
& \text { - Nachträge } \\
& \text { - Beseitigung von Fehlklassierungen } \\
& \text { - Korrektur der Nachbarschaftsverhältnisse } \\
& \text { - Korrekturen der Seenumrisse. }
\end{aligned}
$$

\section{Korrekturen der Gemeindegrenzen}

\section{Beseitigung von Kartenfehlern}

Das Merkmal «Gemeindezugehörigkeit» wurde ursprünglich für jede einzelne Hektare durch Auflegen einer Rasterfolie aus der Landeskarte 1:25000 bestimmt (MEYER 1982, 312). Zum Zeitpunkt dieser Aufnahme (Abschluß der Arbeiten 1972) war das Landeskartenwerk - v. a. im Berggebiet - noch nicht vollständig verfügbar. In diesen Gebieten wurde deshalb auf die Landeskarte 1:50000 zurückgegriffen. Manche Kartenblätter waren damals erst einmal erschienen und enthielten noch Fehler. Besonders häufig traten solche an den Rändern der Kartenblätter auf. Vereinzelt fehlten auf der Landeskarte zudem ganze Grenzlinienabschnitte. In diesen Fällen dienten für das Hektarraster offensichtlich natürliche Grenzen (Gratlinien, Bäche) als Ersatz. Weil dabei kaum je das benachbarte Kartenblatt zu Rate gezogen wurde, folgen die Gemeindegranzen im Hektarraster oft stellenweise dem Kartenblattrand (vgl. Abb. 3). Diese Kartenfehler wurden bei der Aufnahme in das Hektarraster mit übernommen. Da die Gemeindegrenzkarte der Schweiz auch in der neuesten Ausgabe (1981) noch mehrere Fehler enthält, wurden alle
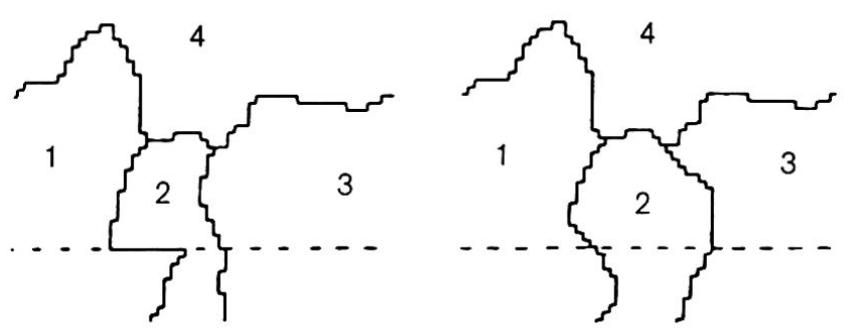

links: Gemeindegrenzen im Hektarraster rechts: Gemeindegrenzen nach der Korrektur --- Kartenblattrand

1 Icogne 2 Randogne 3 Mollens 4 Lenk

Abb.3: Kartenfehler an der Schnittstelle zweier Kartenblätter 
Abweichungen zwischen dem Hektarraster und der Gemeindegrenzkarte 1981 sorgfältig mit dem Stand der neuesten verfügbaren Landeskarte 1:25000 verglichen. Bei Abweichungen zwischen diesen zwei Kartenwerken wurde der Situation auf der Landeskarte Vorrang gegeben (4).

\section{Nachträge}

In der Schweiz sind nicht alle Hoheitsgrenzen rechtsverbindlich definiert (MEYER 1982, 313). Seit der Aufnahme des Hektarrasters kam es zu zahlreichen Änderungen im Verlauf der Gemeindegrenzen. Zu erwähnen sind hier in erster Linie Grenzabschnitte, die infolge einer erstmaligen oder neuen Ausmarchung einen anderen Verlauf erhielten. Im weiteren fanden in der Zwischenzeit zahlreiche Gebietsabtausche zwischen Gemeinden sowie einseitige Gebietsabtretungen infolge von Verkehrsbauten statt. Durch Nachführungen dieser Art ergaben sich teilweise völlig veränderte Gemeindeumrisse (vgl. Abb.4). Inzwischen eingetretene Gemeindefusionen bzw. -spaltungen wurden ebenfalls nachgeführt. Elimination von Kartenfehlern und Nachträge führten zur Korrektur von ca. $400 \mathrm{Gemeindegrenzabschnitten.}$

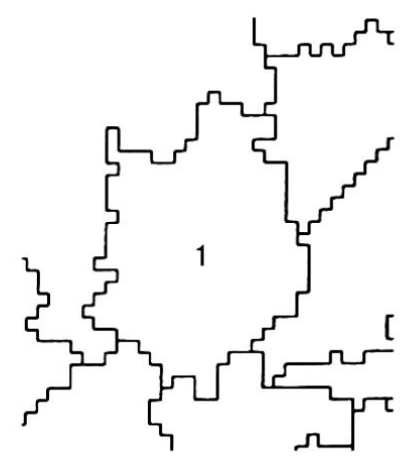

links: Gemeindegrenzen im Hektarraster rechts: Gemeindegrenzen nach der Korrektur

\section{Beseitigung von Fehlklassierungen}

Fehlklassierungen sind Fehler im Hektarraster, die ihren Ursprung nicht in der Landeskarte haben. Meistens handelt es sich um Gebiete mit einer Fläche von weniger als 20 ha, die übersehen wurden. Im Berggebiet führte das Übersehen von Grenzlinienabschnitten in einigen Fällen allerdings zu Abweichungen von mehreren Quadratkilometern. $\mathrm{Ab}$ und $\mathrm{zu}$ wurde der Grenzlinienverlauf zwar richtig aus der Karte übertragen, aber das entsprechende Gebiet mit einer falschen Gemeindenummer codiert.

\section{Korrektur der Nachbarschaftsverhältnisse}

Die größte Anzahl Änderungen - allerdings zumeist nur je eine Hektare umfassend - betraf die Korrektur der Nachbarschaftsverhältnisse. Im Interesse möglichst idealer Linienformen mußte vom ursprüngli- chen Dominanzprinzip des Informationsrasters abgerückt werden. Maßgeblich für die Zuordnung jeder einzelnen Hektare war nun nicht mehr der größte Flächenanteil, sondern ein möglichst zutreffendes Abbild der Nachbarschaftsverhältnisse. Durch sorgfältigen Vergleich mit der Landeskarte 1:25000 konnte sichergestellt werden, daß nur dann vier Gemeindegrenzlinien zusammenlaufen, wenn wirklich vier Gemeinden an einem Punkt zusammenstoßen. Alle Grenzknoten wurden deshalb überprüft. Damit wurde erreicht, daß in der korrigierten Hektarraster-Datei nie zwei Gemeinden aneinanderstoßen, die nicht auch in Wirklichkeit eine gemeinsame Grenzlinie besitzen. Auf diese Weise wurden alle «künstlichen Exklaven» beseitigt, so daß zusammenhängende Gebiete auch in der Raster-Datei eine zusammenhängende Fläche bilden. Besonders in NW-SE- oder SW-NE-Richtung verlaufende schmale Korridore wurden der Realität entsprechend in eine zusammenhängende Fläche übergeführt (vgl. Abb. 5). Dadurch wurde die Zahl der Arcs in der Grenzliniendatei um ca. $10 \%$ reduziert.

Die Änderungen betrafen meist nur wenige Hektaren, in Einzelfällen aber mehrere Quadratkilometer. Bei den großflächigen Änderungen kamen die Vorzüge der oben beschriebenen Editier- und Korrekturprogramme voll zur Geltung, indem nur die neue Grenzlinie Hektare für Hektare eingegeben werden mußte, die dabei neu entstandene Innenfläche jedoch gesamthaft der neuen Gemeinde zugeordnet werden konnte.

Insgesamt erfaßte die Korrektur der Gemeindezugehörigkeit 15414 Hektaren; 1038 Gemeinden erhielten einen anderen Grenzverlauf.

\section{Korrekturen der Seenumrisse}

Bei Karten der Schweiz ist es üblich, auch in kleineren Maßstäben als Orientierungshilfe zumindest die gröBeren Seen abzubilden. Um aus dem Hektarraster die Seenumrisse zu übernehmen, mußte auch die Bodennutzung (stehende Gewässer - übriges) überprüft werden. Alle Seen mit einer Gesamtfläche von über 30 Hektaren wurden mit einem ihrer Fläche entsprechenden Code versehen. Bei der Generierung der Grenzen können so - je nach der Aufgabenstellung und dem angestrebten Generalisierungsgrad - die Seen gewünschter Größe miteinbezogen werden. Wie bei den Nachführungen der Gemeindegrenzen wurden auch hier Änderungen nachgetragen (z. B. neue Stauseen). Analog zur Korrektur der Gemeindegrenzen wurden die Umrißlinien der Seen so überarbeitet, $\mathrm{da} ß$ sie zusammenhängende Uferlinien bilden.

\section{Die Umwandlung des Datenrasters in Umrißvektoren}

Es existieren bereits andere Programme, welche aus Rasterdateien Grenzvektoren heraussuchen (z.B. BRASSEL 1979, NICHOLS 1982, LOGAN und WOODCOCK 

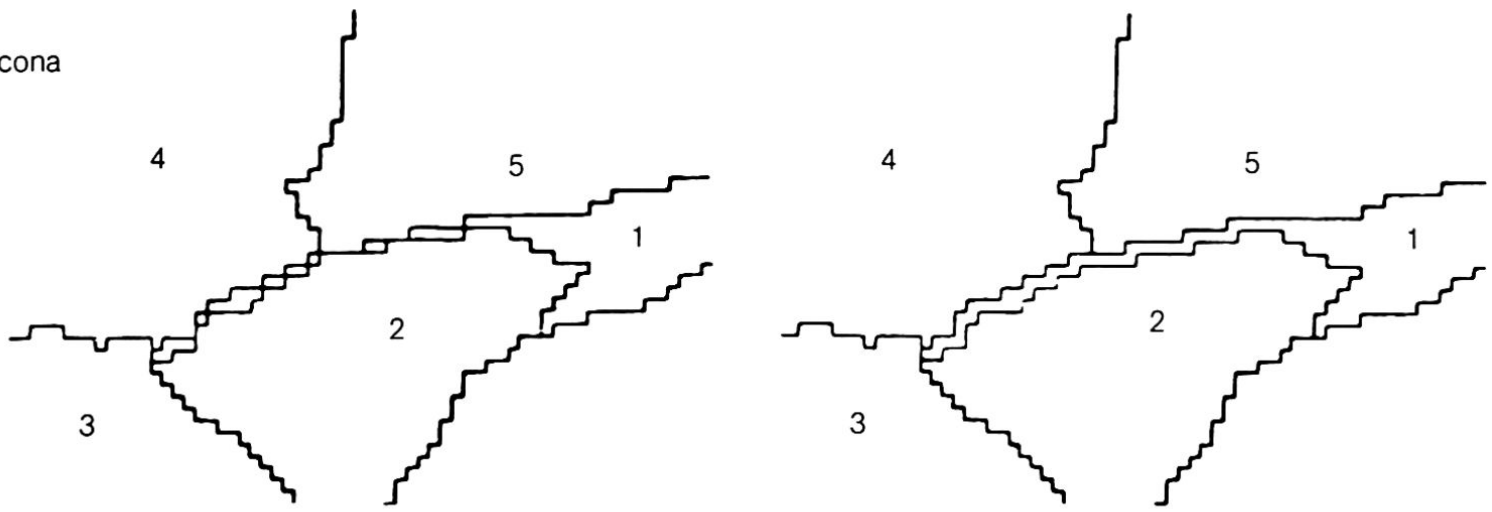

links: Gemeindegrenzen im Hektarraster rechts: Gemeindegrenzen nach der Korrektur

Abb. 5: Beseitigung der durch die Rasterform bedingten Zerstückelung von schmalen Gebietskorridoren

1983). Hier wurde eine Prozedur entwickelt, die den speziellen Anforderungen an die resultierende Datei möglichst gerecht werden kann.

Die Verfolgung der Grenzen erfolgt durch das Wandern eines $2 \times 2$-Feldes über ein aus dem Raster entnommenes Teilgebiet (vgl. auch MONMONIER 1982, 81f.). Die Nachbarschaftsverhältnisse der vier Zellen legen eindeutig fest, in welcher Richtung weitergewandert werden muß. Dieses kleine Feld dient auch zur Erkennung von Knoten: Abbildung 6 zeigt die sechs möglichen Fälle von Feldern, die bei der Wanderung entlang einer Grenze auftreten können (abgesehen von Symmetrien und Rotationen). Die vier Felder sind ihrem Flächencode entsprechend mit unterschiedlichen Signaturen bezeichnet. Der Fall 1 definiert einen Eckpunkt, der Fall 2 einen Kantenpunkt. Die Fälle 3 bis 6 beschreiben Knoten; Fall 3 könnte allenfalls als normaler Grenzpunkt definiert werden; wird er aber als Knoten erfaßt, so sind die Grenzverläufe eindeutig festgelegt.

Zunächst wird in jeder Rasterzelle der Gemeindecode durch einen Regionscode ersetzt (5). Fällt eine Rasterzelle auf einen See, der in der Grenzliniendatei berücksichtigt werden soll, so wird dieser Zelle der Code "See» zugewiesen. Die minimale Seengröße ist ja nach Aggregationsniveau der Gemeinden verschieden anzusetzen.

Das Auffinden der Grenzlinien erfolgt regionsweise. Vorerst werden alle Zellen des umschließenden Rechtecks der Region in den Hauptspeicher eingelesen, darin wird ein erster Grenzknoten gesucht. Von dort aus wird mit Hilfe des 2 x 2-Feldes der Grenzverlauf abgetastet und Grenzkette um Grenzkette in der Vektorendatei abgelegt. Besteht eine Region aus mehreren Teilpolygonen oder besitzt sie Enklaven, so muß für jede geschlossene Umrißlinie ein erster Grenzpunkt gefunden und von dort aus die Grenzlinie verfolgt werden.
Die resultierende Grenzliniendatei besteht aus der Beschreibung einer Anzahl von Grenzketten; Anfangs- und Endpunkte der einzelnen Grenzstücke sind jeweils Knoten. Ausnahmen bilden lediglich gewisse Exklaven bzw. Enklaven, Inseln und Seen, deren Gebiet vollständig von einer einzigen Gebietseinheit umschlossen wird. In diesen Fällen wird willkürlich ein Punkt der Grenzlinie zum Anfangsund Endpunkt gemacht.
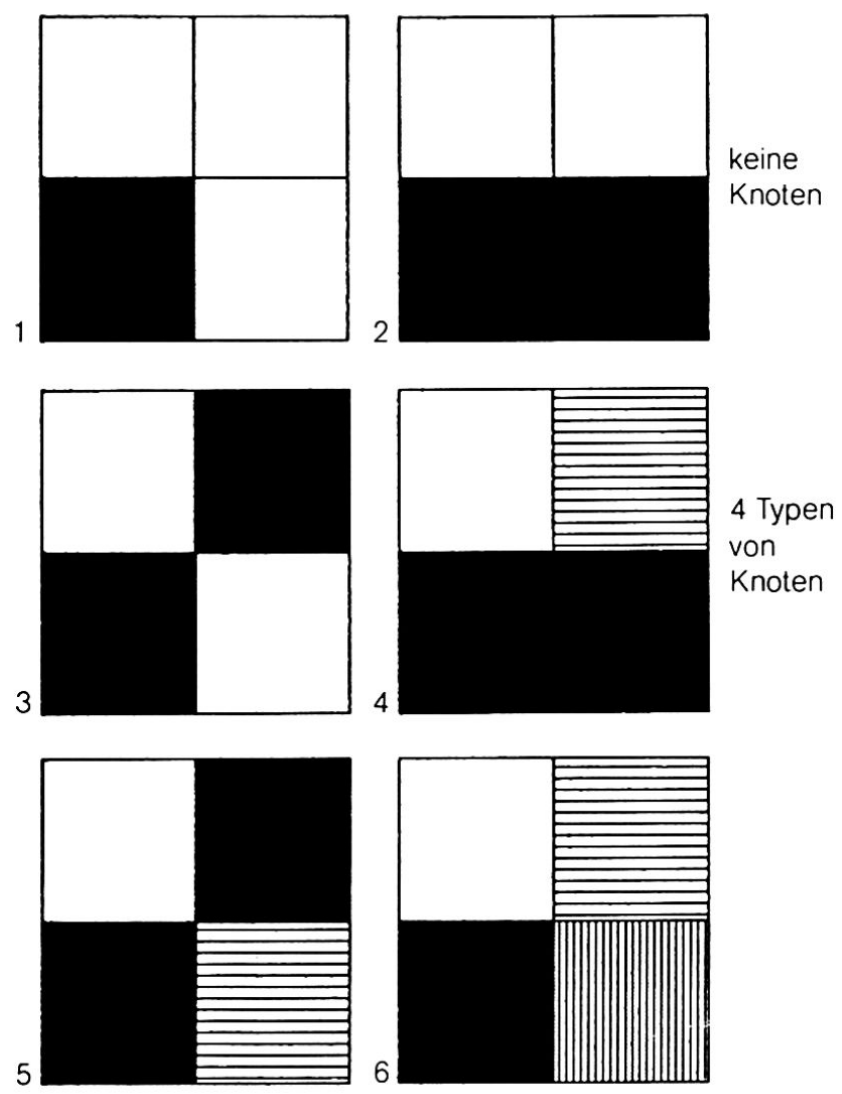

Abb. 6: Die Definition des Knotens im Raster 
Tabelle 1 Die Grenzdateien verschiedener administrativer Einheiten

\begin{tabular}{lrrrr}
\hline Gebietseinheiten & Gemeinden & Bezirke & Planungsregionen & MS-Regionen \\
\hline Zahl Gebiete & $3036 \bullet$ & 184 & 143 & 106 \\
Arcs & 18481 & 1251 & 963 & 735 \\
Arcs/Gebiet & 6.09 & 6.80 & 6.73 & 6.93 \\
Punkte & 410003 & 125746 & 112269 & 100410 \\
Punkte/Arc & 22.19 & 100.52 & 116.58 & 136.61 \\
Punkte/Gebiet & 135.0 & 683.4 & 785.1 & 947.3 \\
Grenzlänge $(\mathrm{km})$ & 63364.8 & 20611.0 & 18377.2 & 16517.8 \\
Länge/Gebiet (km) & 20.87 & 112.02 & 128.51 & 155.83 \\
Länge/Arc $(\mathrm{km})$ & 3.43 & 16.48 & 19.08 & 22.47 \\
max. Pt./Arc & 292 & 806 & 814 & 1271 \\
max. Arclänge (km) & 53.3 & 122.4 & 146.7 & 15 \\
max. Arc/Geb. & 24 & 17 & 206.1 \\
\hline
\end{tabular}

- Einschließlich der Communanze des Tessins und des Freiburger Staatswalds Galm

Im übrigen bestehen alle Arcs aus mindestens zwei Punkten, ihre minimale Länge beträgt eine Rastereinheit (hier: $100 \mathrm{~m}$ ). Die einzelnen Segmente der Arcs verlaufen alle exakt in Richtung der Achsen des Rasters (hier: west-, ost-, süd- oder nordwärts), drei aufeinanderfolgende Punkte schließen jeweils einen rechten Winkel ein. Die Grenzlinien haben dadurch ein treppenförmiges Aussehen.

Das Programm liefert zudem einige statistische Angaben über die Grenzliniendateien (vgl. Tabelle 1).

\section{Grenzlinienglättung und -generalisierung}

Treppenartige Grenzlinien beschreiben wohl den exakten Grenzverlauf in einer Rasterdatei, sie entsprechen dem effektiven Grenzverlauf aber kaum. Die hohe Punktzahl in gewissen Abschnitten täuscht eine Scheingenauigkeit vor: Die Übertragung der wirklichen Grenzen ins Raster hat nämlich dazu geführt, daß schräg zur Rasterorientierung verlaufende Geradenabschnitte zu Treppenlinien geworden sind. Diese Treppenlinien sind zudem in großmaßstäbigen Karten ästhetisch unbefriedigend. Aus diesen Gründen wird versucht, die treppenförmigen Grenzlinien an die ihnen zugrundeliegenden wirklichen Grenzen möglichst gut anzugleichen.

Viele Grenzabschnitte können dadurch geglättet werden, daß - abgesehen vom Anfangs- und Endpunkt eines Bogens, die immer erhalten bleiben müssen - jeweils die Mitten der Strecken zwischen zwei Nachbarpunkten als neue Grenzpunkte die alten Treppenpunkte ersetzen. Um zu verhindern, daß zu große Stücke abgeschnitten werden, wird ein Treppenpunkt beibehalten, falls die beiden angrenzenden Strecken je länger als eine Rastereinheit sind. $\mathrm{Da}$ Punkte, die auf einer Geraden liegen, weggelassen werden können, reduziert sich die Menge der Punkte etwa um einen Viertel.
Im Hinblick auf den Rechenaufwand der kartographischen Programme kann anschließend die Datenmenge durch eine Generalisierung der Grenzlinien verkleinert werden. Die Reduktion der Zahl der Grenzpunkte wird hier nach DOUgLas und PEUCKER (1973, 117ff.) vorgenommen. Für die Generalisierung der Bezirke, Planungsregionen etc. wurden die Grenzen vorgängig nicht geglättet, sondern direkt generalisiert. Bei nur geringer Generalisierung führt allerdings der Zwischenschritt der Glättung zu besseren Resultaten.

\section{Das Erstellen von geschlossenen Polygonen}

Um die gewonnenen Grenzliniendateien für die Herstellung von Computerkarten gebrauchen zu können, sind meist geschlossene Polygonumrisse notwendig. Die einzelnen Grenzketten eines Gebiets mußten deshalb zu Polygonzügen zusammengehängt werden. $\mathrm{Zu}$ Beginn dieses Prozesses liegen die Grenzketten vor, für die die beiden Endknoten, der Grenzverlauf und die beiden benachbarten Flächen bekannt sind. Für jeden Knoten wird nun ein Verzeichnis erstellt, das Auskunft über die ankommenden und wegführenden Grenzketten gibt. In dieser Liste werden die Arcs im Uhrzeigersinn geordnet. Dies erlaubt auf einfache Weise, die Polygonzüge zu erstellen: Man findet vorerst eine erste Grenzkette einer Region und folgt dieser bis zum nächsten Knoten, wo dessen Arcverzeichnis dann als eine Art Wegweiser auf die anschließende Grenzkette benützt werden kann.

Diese Prozedur wurde für sämtliche bearbeiteten Regionalisierungen verwendet. Für kleinmaßstäbige Anwendungen ist es möglich, die geglätteten Grenzen zuerst zu generalisieren und erst dann Polygone zu bilden. Dieses Verfahren kann auch für eine beliebige Gruppe von Gemeinden durchgeführt werden. 


\section{Ergebnisse und Anwendungen}

Wir haben ein Verfahren dargestellt, das aufgrund der Merkmale «Gemeindezugehörigkeit» und "Bodennutzung" (Seen) Umrißdateien von Gemeinden oder Agglomerationen von Gemeinden (Bezirke, Planungsregionen, IHG-Regionen (6) etc.) erstellt. In der Regel weichen diese Umrisse nicht mehr als 50 Meter vom wahren Grenzverlauf ab. Die einzelnen Schritte sind anhand eines Ausschnittes aus dem Kanton Zürich veranschaulicht. Abbildung 7a zeigt die Gemeindegrenzen des Informationsrasters, $7 \mathrm{~b}$ die Umrißdatei, die aus dem korrigierten Informationsraster abgeleitet wurde. In Abbildung 7c ist die Umrißdatei geglättet worden. Die Figuren 7d und 7e zeigen Generalisierungen verschiedenen Ausmaßes. Zahl der Punkte für den ganzen Kt. Zürich: $22366(100 \%)$ in der Rohdatei (Abb. 7b), $16118(72,1 \%)$ in der geglätteten Datei (Abb. 7c) und $6086(27,2 \%)$ bzw. $2716(12,1 \%)$ nach der Generalisierung (Abb.7d bzw. 7e). Die erzeugten
Umrißdateien können nun als Grundlagen für verschiedene Kartierpakete verwendet werden. Abbildung 8 zeigt ein Beispiel des Kartierprogrammes COPAM (BRASSEl und utano 1979), mit dem die Gemeinden der IHG-Region Locarno dargestellt wurden. Außer für administrative Einheiten könnte das System im Prinzip auch für die Abgrenzung beliebiger in Rasterform gespeicherter Merkmale verwendet werden. So wäre es z. B. möglich, die Umrisse aller als "Wald" codierten Gebiete, oder die Gebiete mit einer Höhenlage zwischen z. B. 800 und $900 \mathrm{~m}$ in Form von polygonalen Umrissen zu beschreiben. Dabei können diese Umrisse wiederum in jedem gewünschten Ausmaß generalisiert werden. Probleme mit dem vorliegenden System stellen sich insofern, als die Bearbeitung von sehr großen Teilregionen noch sehr viel Speicherplatz beansprucht. Zukünftige Anstrengungen sind notwendig, um den Anwendungsbereich der verwendeten Prozeduren zu verbreitern.

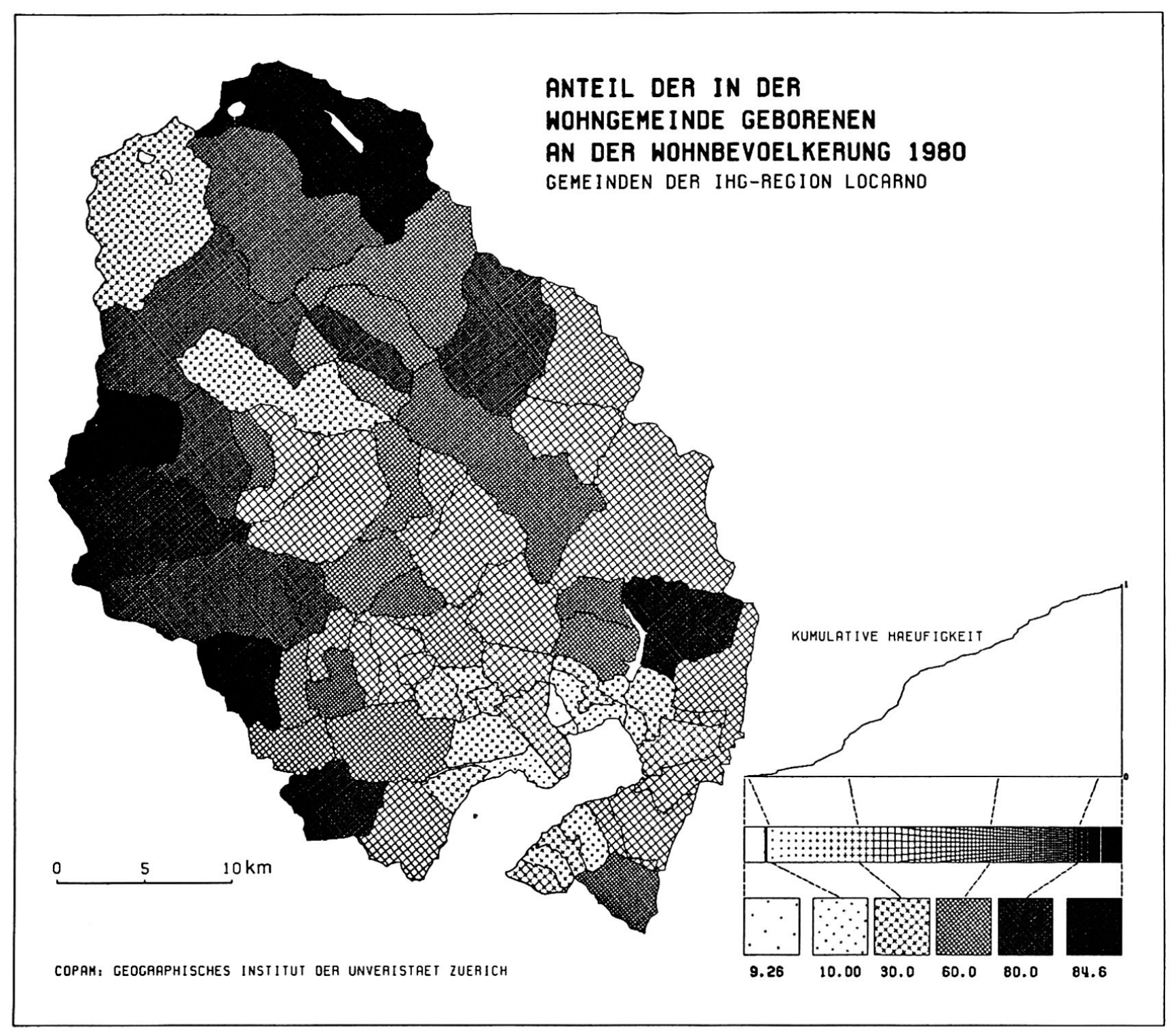

Abb. 8: Beispiel einer COPAM-Karte 
a

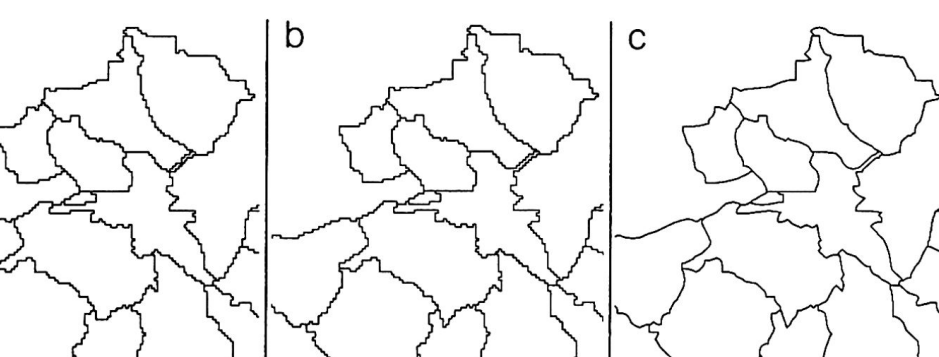

d

(3)

4



Abb. 7: Die einzelnen Umwandlungen am Beispiel eines Ausschnittes aus dem Kt. Zürich 


\section{Anmerkungen}

1 Planungsregionen: Raumplanungsregionen gemäß Bundesamt für Raumplanung. Stand Mai 1981; MS: mobilité spatiale (Regionalisierung nach BASSAND und BRULHARDT 1980)

${ }^{2}$ Karten wurden publiziert in: SCHULER und NEF (1983; 32f., 104, 106, 108, 116ff., 149ff.)

${ }^{3}$ Für nähere Informationen über das Informationsraster sei auf das Benützerhandbuch und auf die Arbeitsberichte zur Orts., Regional- und Landesplanung Nr. 4 (1970) hingewiesen.

\section{Literaturverzeichnis}

BASSAND, Michel und Marie-Claude BRULHARDT (1980): Mobilité spatiale, Nationales Forschungsprogramm Regionalprobleme, Band 5, St-Saphorin.

BRASSEL Kurt (1979): "Shaded Maps from Raster Data." Paper presented at the Forth Annual Meeting of the Canadian Cartographical Association, Toronto May 1979.

BRASSEL Kurt (1983): "Grundkonzepte und technische Aspekte von geographischen Informationssystemen.» In: Internationales Jahrbuch für Kartographie, Band XXIII, Bonn Bad Godesberg.

BRASSEL Kurt und Jack J. UTANO (1979): “Design Strategies for Continuous-tone Area Mapping." In: The American Cartographer, Vol. 6, No. 1, 39-50.

DORIGO, Guido (1983): VCPLOT, Geo-Processing Reihe, Vol. 2, Geographisches Institut der Universität Zürich.

DOUGLAS, David H. und Thomas K. PEUCKER (1973): “Algorithms for the Reduction of the Number of Points Required to Represent a Digitized Line or Its Caricature." In: The Canadian Cartographer, Vol. 10, No. 2, S. 112-122.

HASE, Klaus (1972): "Aufbau des Informationsrasters." In: DISP, Sondernummer Informationsraster, Nr. 24, 7-14.

HERZOG, Adrian, Kurt BRASSEL, Matthias BOPP und Guido DORIGO (1983): Ein System zur Generierung von Grenzliniendateien administrativer Einheiten der Schweiz, Geo-Processing Reihe, Vol. 3, Geographisches Institut der Universität Zürich.

INTERNATIONAL CARTOGRAPHIC ASSOCIATION (1980): Glossary of Terms in Computer Assisted Cartography, Falls Church.

LOGAN, Thomas L. und Curtis E. WOODCOCK (1983): “User Alternatives in Post-Processing for Raster-to-Vector Conversion." In: Proceedings ISPRS Commission IV Symposium 1982 Environmental Assessment and Resource Management, 397-407.

MEYER, B. (1982): “Arealstatistik - Rückblick und Ausblick.» In: Vermessung, Photogrammetrie, Kulturtechnik, Heft 10/82, 310-317.

MONMONIER, Mark S. (1982): Computer-Assisted Cartography, Principles and Prospects, Englewood Cliffs, N. J.

NICHOLS, David A. (1982): "Conversion of Raster Coded Images to Polygonal Data Structures." In: PECORA VII Symposium, Proceedings, 508-515.
${ }^{4}$ Für eine detaillierte Betrachtung sei auf HERZOG et al. (1983) verwiesen, wo auch eine Vielzahl von Beispielen gegeben wird.

${ }^{5}$ Sollen Gemeindegrenzen herausgesucht werden, wird der Gemeindecode als Regionscode verwendet.

${ }^{6}$ Regionen gemäss Investitionshilfe-Gesetz.
PEUCKER, Thomas K. und Nicholas CHRISMAN (1975): "Cartographic Data Structures." In: The American Cartographer, Vol. 2, No. 1, 55-69.

RICHASON, Benjamin F. (Ed.) (1982): PECORA VII Symposium, Proceedings: Remote Sensing: an Input to Geographic Information Systems in the 1980's, Falls Church, Va.

SCHULER, Martin und Rolf NEF (1983): Räumliche Typologien des schweizerischen Zentren-Peripherien-Musters, Nationales Forschungsprogramm "Regionalprobleme in der Schweiz", Arbeitsbericht 35, Bern.

\section{Quellenverzeichnis}

Die Gemeinden der Schweiz, 4 Karten 1:200 000, Ausgabe 1981, hrsg. vom Bundesamt für Landestopographie, Wabern. Informationsraster Benützerhandbuch, hrsg. vom Bundesamt für Statistik und vom Bundesamt für Raumplanung (1980), Arbeitsdokumente für die schweizerische Statistik, Heft 3, Bern. Landeskarte der Schweiz, hrsg. vom Bundesamt für Landestopographie, Wabern.

Statistisches Jahrbuch der Schweiz 1981, hrsg. vom Bundesamt für Statistik, Bern. 